Notre Dame Journal of Formal Logic

Volume 29, Number 3, Summer 1988

\title{
Preliminary Notes on a New Modal Syllogistic
}

\author{
GEORGE ENGLEBRETSEN
}

This article consists of five parts. In Section 1 we introduce the topic of modal syllogistic by examining the case of the two Barbaras found in Prior Analytics. In the second section we briefly review certain aspects of the "new syllogistic" developed in recent years by Fred Sommers. The next two sections examine some of the syntactic and semantic features of modal sentences de dicto and de re respectively. Our final section presents a preliminary sketch of what a syllogistic admitting both de dicto and de re modality would look like.

1 The following dilemma for modal syllogistic was once posed by Martha Kneale:

If modal words modify predicates, there is no need for a special theory of modal syllogisms. For these are only ordinary assertoric syllogisms of which the premisses have peculiar predicates. On the other hand, if modal words modify the whole statements to which they are attached, there is no need for a special modal syllogistic, since the rules determining the logical relations between modal statements are independent of the character of the propositions governed by the modal words. ([7], p. 91)

Syllogistic's author, Aristotle, was clearly aware of the de dicto/de re distinction alluded to here. In De Interpretatione he was at some pains to spell out various laws of modal propositional logic, where modal words are purely de dicto. But in Prior Analytics he attempted a modal syllogistic. The degree of his success in this is still an issue of debate. The question has never been whether or not a modal syllogistic could be formulated. Rather it has been whether one could be formulated which preserves both formal adequacy and what McCall ([9]) called "Aristotelicity". How important it is to preserve all of Aristotle's claims, insights, intuitions, and hunches is itself debatable. There are places in Aristotle's work which are simply muddled. For example, despite the fact that he clearly saw the formal distinction between de dicto and de re sentences he sometimes takes corresponding pairs of de dicto/de re sentences as interchange- 
able. Thus he states in Prior Analytics (25a29) the apodeictic negative universal as ' $A$ necessarily applies to no $B$ ' and (30b12) as ' $A$ possibly applies to no $B$ '. Now the first is easily reformulated as

(1) Necessarily no $B$ is $A$.

The second would appear to be equivalent at first sight to

(2) Possibly no $B$ is A.

But taking (1) and (2) as equivalent is too gross an error to attribute to Aristotle. He surely took the second sentence to be a de re version of the first; that is,

(3) No $B$ is possibly $A$.

Nonetheless, contra McCall, it was an error (though a more subtle one) to equate (1) and (3). For let ' $A$ ' be uniformly replaced by 'non $B$ '. We would then have the following substitutions.

(1.1) Necessarily no $B$ is non $B$

(3.1) No $B$ is possibly non $B$.

Now (1.1) is obviously true ('No $B$ is non $B$ ' is analytic). But (3.1) is just false. Consider: 'No bachelor is possibly a nonbachelor' (='No bachelor is a possible nonbachelor'). Surely Michael Jackson is an actual bachelor who is a possible nonbachelor; it's only a contingent matter that he is a bachelor.

If (1) and (3) are nonequivalent, the semantic as well as formal de dicto/de re distinction must be preserved. And, while Aristotle seems to have blurred the difference here, he must have been recognizing it clearly (if not explicitly) elsewhere. Aristotle's discussion of "the two Barbaras" (Prior Analytics 30a21-28) is a good place to see this. Aristotle is talking there about syllogisms with mixed categorical and apodeictic premises and an apodeictic conclusion. Some are valid, others are not. For example, Barbara with the major and conclusion apodeictic is valid, but Barbara with minor and conclusion apodeictic is invalid. Let us call these "Barbara I" and "Barbara II" respectively. ${ }^{1}$ They are:

Barbara I: $A$ necessarily applies to all $B$

$B$ applies to all $C$

So $A$ necessarily applies to all $C$

Barbara II: $A$ applies to all $B$

$B$ necessarily applies to all $C$

So $A$ necessarily applies to all $C$.

If we interpret the modal words in these as explicitly de dicto we get

Barbara $\mathrm{I}(\mathrm{d})$ : Necessarily all $B$ is $A$

All $C$ is $B$

So necessarily all $C$ is $A$

Barbara II(d): All $B$ is $A$

Necessarily all $C$ is $B$

So necessarily all $C$ is $A$. 
Now Aristotle's thesis is that Barbara I, but not Barbara II, is valid. Does this difference hold for the de dicto readings? Barbara II(d) is invalid. A counterargument would be to substitute $C$ for $B$. Thus:

\author{
All $C$ is $A$ \\ Necessarily all $C$ is $C$ \\ So necessarily all $C$ is A.
}

Here the assertoric/modal (viz. apodeictic) distinction guarantees its invalidity. But a similar counterargument can just as well be used to show the invalidity of Barbara I(d). ${ }^{2}$ Let $A$ be a substitute for $B$, then

Necessarily all $A$ is $A$

All $C$ is $A$

So necessarily all $C$ is $A$.

So let us give the two Barbaras an explicitly de re reading.

Barbara I(r): $\quad$ All $B$ is necessarily $A$

All $C$ is $B$

So all $C$ is necessarily $A$

Barbara II(r): All $B$ is $A$

All $C$ is necessarily $B$

So all $C$ is necessarily $A$.

There is no question that Barbara II(r) is formally invalid. But what of Barbara $\mathrm{I}(\mathrm{r})$ ? Suppose we try to show it invalid by a counterargument parallel to the one used against Barbara I(d). Substituting $A$ for $B$ we get

All $A$ is necessarily $A$

All $C$ is $A$

So all $C$ is necessarily $A$.

Unlike the major of the (d) counterargument, this major is not analytic. There is at least one false instance of it. Consider: 'All bachelors are necessarily bachelors'. Again, Michael Jackson is a bachelor who is a possible nonbachelor. So calling the counterargument valid would not force us to derive the conclusion from the minor alone (in turn collapsing the assertoric/apodeictic distinction). So this is not a counterargument to Barbara I(r). In fact, Barbara I(r) is valid. When de re modal expressions are seen as forming new "peculiar" predicates (i.e., predicate-terms); i.e., 'All $B$ is (necessarily $A$ )' rather than 'All $B$ (is necessarily) $A$ ', the formal validity of Barbara I(r) is guaranteed by the formal validity of Barbara. Thus it is only the de re readings which preserve Aristotle's thesis that Barbara I, but not Barbara II, is valid.

2 A syllogistic of de re modality can be easily constructed. It is simply a fragment of the assertoric syllogistic with modalized terms. But both syllogistic and de re modality have lost favor in this century. Although it has had its recent defenders, de re modality has been denigrated by logicians like Quine who are repelled by what are perceived as its essentialist consequences. And syllogistic 
is even more broadly scorned. At worst it is seen as downright mistaken. At best it is seen as merely a fragment of the standard first-order function calculus.

Quine's worries are caused by the possibility of allowing modal expressions to function within the scope of a quantifier. Such "quantifying in" through modal contexts cannot be permitted since such contexts are referentially opaque. But these worries vanish if both quantifiers and modals are taken as term functors rather than sentence functors. Of course the real trick here is construing quantifiers as term functors. To do so would require an effective logic of terms. Ironically, Quine himself has constructed one version of such a logic: the predicate functor algebra (see [10] through [23]). Another recent version of a term logic is Sommer's "new syllogistic" (see [25] through [28] and [6]).

Sommers's logic deals with the canonical fragment of a natural language whose sentences either have a finitely and recursively specifiable syntax, or can be paraphrased as sentences which do. The canonical sentences are logically categorical, consisting of a subject and a predicate. A subject is a syntactically complex expression consisting of a quantifier and a term. A predicate is a syntactically complex expression consisting of a qualifier (copula) and a term. Terms are either syntactically simple or complex. A complex term is syntactically categorical. On this theory quantifiers are not functors operating on entire sentences. Instead they are functions on terms, yielding subjects. Quantifiers are universal or particular. Qualifiers are affirmative or negative. And terms come in positive/negative pairs. For example, 'red' and 'nonred' are such a pair.

The elementary sentences of Sommers's syllogistic are particular affirmations and their corresponding negatives (viz. contradictories). Letting $S$ and $P$ be any terms, the following represent elementary sentences.

1. An $S$ is $P$

2. An $S$ is non $P$

3. Not an $S$ is $P$ (No $S$ is $P$ )

4. Not an $S$ is non $P$ (No $S$ is non $P$ ).

Part of Sommers's logic is a symbolic algorithm which achieves maximum efficacy with a minimum of symbolic devices. All particular quantifiers are reformulated by +'s. Affirmations are indicated by a + preceding the formula. Negations are likewise indicated by -'s, which are also used to indicate negated terms. Positive terms are marked + . The sentences above are then symbolized as follows (where parentheses are, as usual, used to resolve questions of scope).

$$
\begin{aligned}
& 1.1+(+(+S)+P) \\
& 2.1+(+(+S)-P) \\
& 3.1-(+(+S)+P) \\
& 4.1-(+(+S)-P)
\end{aligned}
$$

As in algebra, we can safely simplify these by supressing initial +'s as well as the signs on positive subject terms. Thus:

$$
\begin{aligned}
& 1.2+S+P \\
& 2.2+S-P \\
& 3.2-(+S+P) \\
& 4.2-(+S-P)
\end{aligned}
$$


Notice that now the first sign in 1.2 and 2.2 is a sign of quantity not quality (since these have no explicit quality sign they are understood as affirmations). Quantity signs are never suppressed.

Now, if we take the universal quantifier to be defined in terms of the particular ${ }^{3}$ and symbolize it accordingly (by - ), we have

$$
\begin{aligned}
& 1.3+S+P \\
& 2.3+S-P \\
& 3.3-S-P \\
& 4.3-S+P .
\end{aligned}
$$

Here all of the initial signs are signs of quantity and all sentences are taken affirmatively. Sentences 3.3 and 4.3 are achieved simply by distributing the quality signs (the initial -'s) in 3.2 and 4.2. We now read 4.3, for example, as 'Every $S$ is $P$ ?.

Singular sentences, those with singular terms as subjects, are taken to be implicitly particular in quantity. They differ from other particulars only in that, given our extralogical knowledge of their singularity, we can infer from them their corresponding universals. ${ }^{4}$

It is easy to imagine how we might add to Sommers's system a "new modal syllogistic". At least, we can formulate modal sentences and specify, where necessary, additional rules of inference. We could add to our lexicon a modalized version of each term per se. Thus, if ' $P$ ' is a term, then ' $\nabla P$ ' (read 'possibly $P$ ' or 'a possible $P$ ') is a term. Such modalized terms can themselves be negative as well as positive, so that we might have expressions of the form ' $-\diamond P$ ', etc. Finally, we could permit terms of any sort (e.g., positive or negative) to be modified by modality, resulting, for example, in such expressions as ' $\diamond-P$ ' and ' $-\diamond-P$ '. By doing so we can take one sign of modality, say 'possibly' $(\diamond)$ as primitive and define necessity ( $\square$ ) accordingly.

Inferences in such a system would be governed by the laws of immediate inference and the syllogism, augmented by laws such as

$$
\begin{aligned}
& \text { If }+S+\square P \text { then }+S+P \\
& \text { If }-S+\square P \text { then }-S+P \\
& \text { If }+S+P \text { then }+S+\diamond P \\
& \text { If }-S+P \text { then }-S+\diamond P .
\end{aligned}
$$

These brief remarks should be sufficient to show that, given the viability of Sommers's term logic, a formally adequate modal syllogistic could be devised.

3 Statements (statement-making sentences) are always made relative to some specifiable domain of discourse (what Sommers sometimes calls the statements' amplitude). Domains are coherent totalities specified by their mutually compatible constituents. Sets, whose members are always fixed, and worlds, whose memberships may vary, are both kinds of domains (see [27], [28], and [4]). We say, 'A black horse won the Kentucky Derby in 1984', and our statement is made relative to the actual world. It is the way the actual world is which determines the truth or falsity of our claim. But we also say, 'A winged horse was captured by Bellerophon', in which case our domain is clearly not the actual world, but rather the world of Greek mythology. I can assert 'There are winged horses' and also 'There are no winged horses' without contradiction as long as my two state- 
ments are not both made relative to a common domain. A logical relation between two statements requires that both can be made relative to a common domain.

On Sommers's theory, to be is to be a constituent of the domain in question. Any canonical statement can be read as an implicit claim that some kind of thing exists or does not exist in some given domain. To say, with respect to the actual world, that some horse is black is implicitly to claim that there are black horses in the actual world. To say, with respect to the mythological world, that some horses are winged is implicitly to claim that there are winged horses in the world of mythology. To say, with respect to the actual world, that no horses are winged is to claim implicitly that there are no winged horses in the actual world. And to say, with respect to the actual world, that every horse is tame is to claim implicitly that there are no wild (=nontame) horses in the actual world. Such implicit claims specify, in effect, the truth conditions for these statements. In general, every statement has both a denotation and a signification (see [28] and [4]). What a statement denotes is the domain relative to which it is used. What a statement signifies is a property of that domain -in particular, what Sommers calls a constitutive property. To say of anything that it has the constitutive property of being $P$-ish is to say that it has a $P$-thing (some thing which is $P$ ) as a constituent. A soup which has salt in it has the constitutive property of being salty (saltish). A domain which has a dog in it has the constitutive property of being dogish. More generally, to use 'An $S$ is $P$ ' relative to domain $d$ to make a statement is to characterize $d$ as having the property of being $S P$-ish (written ' $[S P]$ '); i.e., as having as one of its constituents an $S$ which is $P$. The implicit truth claim of any statement is that the domain which it denotes (viz. the one relative to which it is used) has the (constitutive) property it signifies.

All canonical statements are made relative to a determinate domain. But some noncanonical statements are made with respect to no determinate domain. These statements are made, we might say, relative to the domain of domains. To this group belong all statements of de dicto modality. For example, the statement 'Possibly some $S$ are $P$ ' does not claim that $S$ 's which are $P$ ( $S P$-things) are in the actual world. Nor does it claim that $S P$-things are possibly in the actual world. It is a statement made with respect not just to the actual world, or any other particular domain, but rather with respect to all domains. (In [27] Sommers calls this "unrestricted amplitude".) It claims implicitly that $S P$-things exist in some (undetermined) domain. Used to make a statement, 'Necessarily some $S$ and $P$ ' claims that $S P$-things are in every domain.

Let $\cup d$ represent the union of all domains, and $\cap d$ represent the intersection of all domains. Since de dicto apodeictic statements make a truth claim about every domain we will say that they are implicitly made relative to $\cap d$. Since de dicto problematic statements make a truth claim about some unspecified domain(s) we will say that they are implicitly made relative to $\cup d$. Letting $[p]$ be the constitutive property signified by ' $p$ ' when used to make a statement, we can say that ' $\square p$ ' ('necessarily $p$ ') implicitly makes the truth claim that every domain is $[p]$; i.e., $\cap d$ is $[p]$. Also ' $\forall p$ ' ('possibly $p$ ') implicitly makes the truth claim that some domain is $[p]$; i.e., $\cup d$ is $[p]$.

Suppose I state that it is necessary that not $p$ (by using ' $\square-p$ '). My implicit truth claim is that every domain lacks the property $[p]$; in other words, no 
domain is $[p], \cap d$ is un $[p]$. We display in Table 1 the eight de dicto modalized categoricals along with their truth claims (' $\bar{P}$ ' means 'non $P$ ').

Table 1.

\begin{tabular}{cl}
\hline Formula & Truth claim \\
\hline$\square \mathrm{a}: \square(-S+P)$ & $\cap d$ is un $[S \bar{P}]$ \\
$\square \mathrm{e}: \square(-S-P)$ & $\cap d$ is un $[S P]$ \\
$\square \mathrm{i}: \square(+S+P)$ & $\cap d$ is $[S P]$ \\
$\square \mathrm{o}: \square(+S-P)$ & $\cap d$ is $[S \bar{P}]$ \\
$\diamond \mathrm{a}: \diamond(-S+P)$ & $\cup d$ is un $[S \bar{P}]$ \\
$\diamond \mathrm{e}: \diamond(-S-P)$ & $\cup d$ is un $[S P]$ \\
$\diamond \mathrm{i}: \diamond(+S+P)$ & $\cup d$ is $[S P]$ \\
$\diamond \mathrm{o}: \diamond(+S-P)$ & $\cup d$ is $[S \bar{P}]$ \\
\hline
\end{tabular}

Note that the contradictory of $\square \mathrm{a}$ is not $\square$ o but rather $\diamond_{\mathrm{o}}$. Indeed, $\square \mathrm{a}, \square \mathrm{e}$, $\diamond \mathrm{i}$, and $\diamond_{0}$ form a genuine square of opposition (see Figure 1).

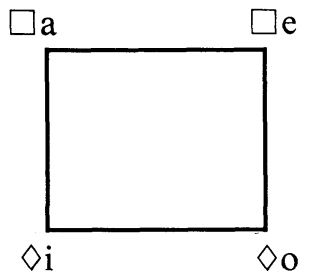

Figure 1

Given the following as axioms: ${ }^{5}$

\section{Axiom 1 If $\square x$ then $x$}

Axiom 2 If $x$ then $\diamond x$

we can display the relations among our twelve assertoric and de dicto modal categoricals as shown in Figure 2 (where the arrow marks implication).

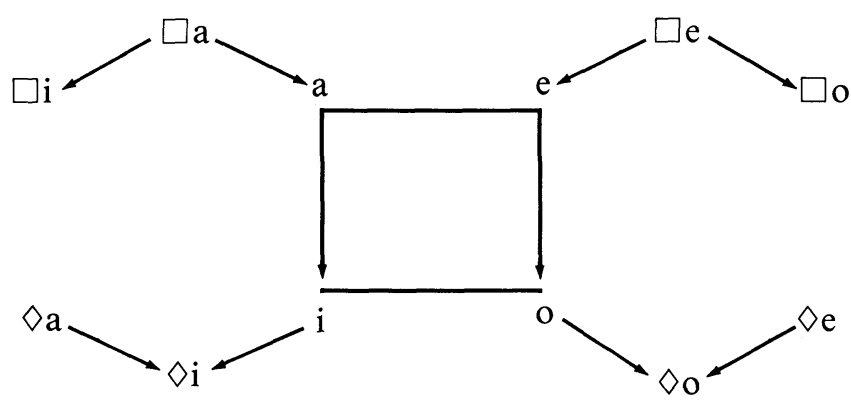

Figure 2 
$4 \quad$ Unlike statements of de dicto modality, statements of de re modality are always made relative to a determinable domain (normally the actual world). Initially we can say that a statement of the form 'Some $S$ is possibly $P$ ', made relative to the actual world, simply claims that there are $S$ 's which are possibly $P$ in the actual world. For example, 'Some horses might have wings', made relative to the actual world, is first paraphrased as 'Some horses are possibly winged'. This statement implicitly claims that some possibly winged horses are constitutents of the actual world. Our next step is to analyze the de re modality which persists here. When I state, relative to the actual world, that some horses are possibly winged I do not implicitly claim that the actual world has any winged horses. What I do implicitly claim is that there is some domain which has as constituents the (possibly winged) horses which are in the actual world, and that these horses are winged in that other domain. Let us say that any domain which has as constituents the constituents of a given domain $d$ is accessible from $d$ ( $d$ is accessible to such a domain). If $d$ is a domain, let $d^{*}$ be a domain accessible from $d$. Now let $\cup d^{*}$ be the union of domains accessible from $d$, and let $\cap d^{*}$ be the intersection of domains accessible from $d$. We can say: $d \subseteq d^{*}$. Accessibility is reflexive and transitive. Now, to state 'Some horses are possibly winged', relative to the actual world, $\alpha$, is to claim implicitly that some domain accessible from $\alpha$ has a winged horse as a constituent.

Generally, to make a de re problematic statement relative to a domain $d$ is to claim implicitly that $\cup d^{*}$ has a specified (constitutive) property. To make a de re apodeictic statement relative to $d$ is to claim implicitly that $\cap d^{*}$ has a specified (constitutive) property. We display in Table 2 the eight de re modalized categoricals along with their truth claims (where the relevant domain is $d$ ).

Table 2.

\begin{tabular}{cl}
\hline \multicolumn{1}{c}{ Formula } & \multicolumn{1}{c}{ Truth claim } \\
\hline $\mathrm{a} \square:-S+\square P$ & $\cap d^{*}$ is un $[S \bar{P}]$ \\
$\mathrm{e} \square:-S+\square-P$ & $\cap d^{*}$ is un $[S P]$ \\
$\mathrm{i} \square:+S+\square P$ & $\cap d^{*}$ is $[S P]$ \\
$\mathrm{o} \square:+S+\square-P$ & $\cap d^{*}$ is $[S \bar{P}]$ \\
$\mathrm{a} \diamond:-S+\diamond P$ & $\cup d^{*}$ is un $[S \bar{P}]$ \\
$\mathrm{e} \diamond:-S+\diamond-P$ & $\cup d^{*}$ is un $[S P]$ \\
$\mathrm{i} \diamond:+S+\diamond P$ & $\cup d^{*}$ is $[S P]$ \\
$\mathrm{o} \diamond:+S-\diamond-P$ & $\cup d^{*}$ is $[S \bar{P}]$ \\
\hline
\end{tabular}

Notice in the table that the contradictory of $a \square$ is not o $\square$ but o $\diamond$. The $a \square$, $\mathrm{e} \square$, $\mathrm{i} \diamond$, and- $\diamond \diamond$ formulas form a genuine square of opposition (see Figure 3).

Given the following axioms:

Axiom 3 If $x \square$ then $x$

Axiom 4 If $x$ then $x \diamond$

we can display the relations among our twelve assertoric and de re modal categoricals as shown in Figure 4.

An examination of Tables 1 and 2 shows that, given that $\cup d^{*} \subseteq \cup d$, and that $\cap d \subseteq \cap d^{*}$ (since, in general, $A \subseteq A \cup B$ and $A \cap B \subseteq A$ ), all de dicto 


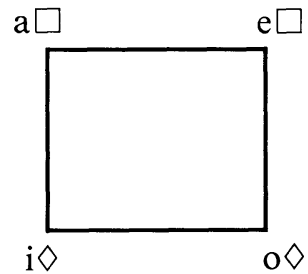

Figure 3

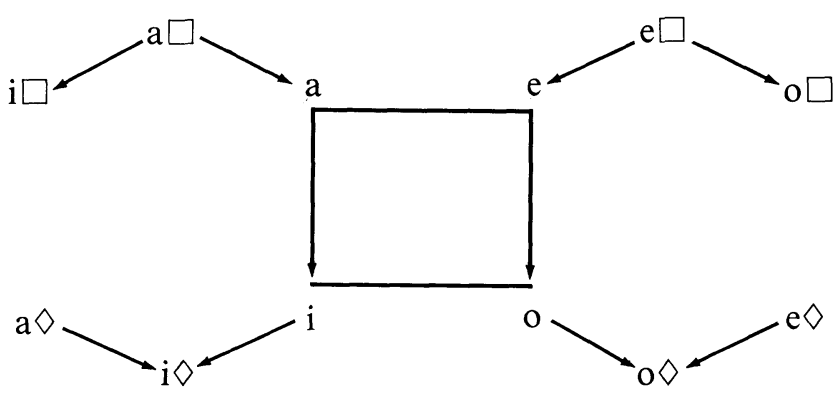

Figure 4

apodeictic statements entail their corresponding de re apodeictic statements, and all de re problematic statements entail their corresponding de dicto problematic statements. In other words,

If $\square x$ then $x \square$

If $x \diamond$ then $\diamond x$.

This means we could combine Figures 2 and 4 to get Figure 5, which displays all the relations among our twenty assertoric and modalized categoricals. ${ }^{6}$

5 The number of assertoric syllogistic forms is quite large. But only about two dozen are actually valid. When we allow the modalization of terms and sentences these numbers increase dramatically. Restricting ourselves to syllogisms consisting only of sentences which are either assertoric or of de dicto modality, the number of syllogisms for each mood is twenty-seven. This is also the number when the modality is restricted to de re. So the total number of kinds of syllogisms for each mood is fifty-three. Of these several hundred syllogistic forms fewer than half are actually valid. ${ }^{7}$ Further syllogistic forms could be generated by permitting a mixture of de re and de dicto modality within the same syllogism. Ockham claimed that by allowing such forms the number of valid moods is about one thousand (see [1], pp. 229-230). In this section we will look at the modalized Barbaras, specify some rules of proof, prove some of the valid Barbaras and, finally, lay down the conditions of validity for a modal syllogism.

The number of Barbaras with sentences which are either assertoric or of de re modality is twenty-seven. Twelve of these are valid. They are: 


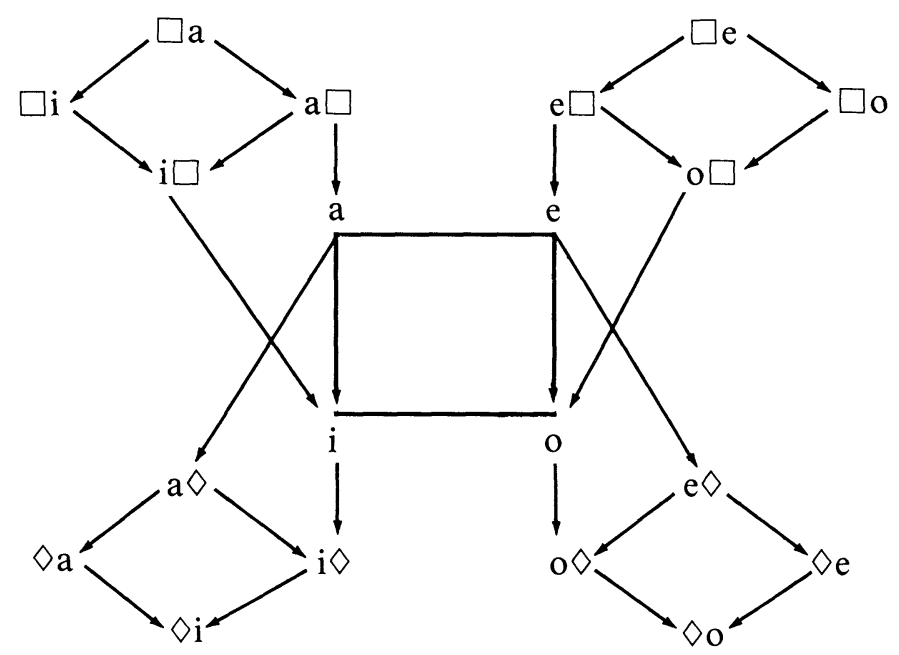

Figure 5

$$
\begin{aligned}
& \text { 1.r } \quad-M+\square P \\
& \text { 2.r } \quad-M+\square P \\
& \text { 3.r }-M+\square P \\
& \frac{-S+\square M}{-S+\square P} \\
& \frac{-S+\square M}{-S+P} \\
& \frac{-S+\square M}{-S+\diamond P} \\
& \text { 4.r } \quad-M+\square P \\
& \text { 5.r }-M+\square P \\
& \frac{-S+M}{-S+\square P} \\
& \frac{-S+M}{-S+P} \\
& \text { 6.r } \quad-M+\square P \\
& \frac{-S+M}{-S+\diamond P} \\
& \text { 7.r } \quad-M+\diamond P \\
& \text { 8.r } \quad-M+P \\
& \frac{-S+\square M}{-S+\diamond P} \\
& \frac{-S+\square M}{-S+P} \\
& \text { 9.r } \quad-M+P \\
& \frac{-S+\square M}{-S+\diamond P} \\
& \text { 10.r } \quad-M+P \\
& \text { 11.r }-M+P \\
& \frac{-S+M}{-S+P} \\
& \frac{-S+M}{-S+\diamond P} \\
& \begin{array}{ll}
12 . \mathrm{r} & -M+\diamond P \\
& \frac{-S+M}{-S+\diamond P}
\end{array}
\end{aligned}
$$

Note that 10.r is the standard Barbara, and 4.r is Barbara I(r) (cf. Section 1 of this essay).

Proofs of the validity of each of these twelve moods requires, first, the usual rule of syllogistic inference-the dictum de omni. This rule, which demands that what is predicated of a distributed term is predicated of what that term is affirmed of, amounts, in Sommers's system, to the requirement that the algebraic sum of the premises must be equivalent to the conclusion. Secondly, Axioms 3 and 4 (Section 4 above) are required. Here are proofs for the first three syllogisms.

Proof of 1.r: 1. $-M+\square P$

premise

2. $-S+\square M$ premise

3. $-S+M$

2, Axiom 3

4. $-S+\square P$ 
$\begin{aligned} & \text { Proof of 2.r: } \text { 1. }-M+\square P \\ & \text { 2. }-S+\square M \\ & \text { 3. }-M+P \\ & \text { 4. }-S+M \\ & \text { 5. }-S+P\end{aligned}$

Proof of 3.r: 1. $-M+\square P$

2. $-S+\square M$

3. $-S+M$

4. $-S+\square P$

5. $-S+P$

6. $-S+\diamond P$ premise premise

1, Axiom 3

2, Axiom 3

3, 4, dictum de omni

premise premise

2, Axiom 3

1, 3, dictum de omni

4, Axiom 3

5, Axiom 4

It is easy to see that the logic of syllogisms containing one or more de re sentences is a simple extension (i.e., the addition of Axioms 3 and 4) of the standard assertoric syllogistic. As Kneale saw, these syllogisms simply have some special terms in them. The overall categorical forms remain. But the admission of de dicto modality into syllogisms requires a slightly more substantial extension of the assertoric syllogistic.

Of the twenty-seven Barbaras with sentences which are either assertoric or de dicto in modality only nine are valid. They are:

$$
\begin{aligned}
& \text { 1.d } \begin{aligned}
& \square(-M+P) \\
& \frac{\square(-S+M)}{\square(-S+P)}
\end{aligned} \\
& \text { 2.d } \\
& \begin{array}{l}
\square(-M+P) \\
\square(-S+M) \\
\hline-S+P
\end{array} \\
& \text { 3.d } \square(-M+P) \\
& \frac{\square(-S+M)}{\diamond(-S+P)} \\
& \text { 4.d } \square(-M+P) \\
& \text { 5.d } \square(-M+P) \\
& \frac{-S+M}{-S+P} \\
& \frac{-S+M}{\nabla(-S+P)} \\
& \text { 6.d }-M+P \\
& \frac{\square(-S+M)}{-S+P} \\
& \text { 7.d } \quad-M+P \\
& \frac{\square(-S+M)}{\diamond(-S+P)} \\
& \begin{array}{cl}
\text { 8.d } & -M+P \\
& -S+M \\
\hline-S+P
\end{array} \\
& \begin{array}{ll}
9 . \mathrm{d} & -M+P \\
& \frac{-S+M}{\diamond(-S+P)}
\end{array}
\end{aligned}
$$

Notice again that 8.d = 10.r (=the standard Barbara). Also note that while 4.r (=Barbara I(r)) was valid, Barbara $\mathrm{I}(\mathrm{d})$ is not among our nine valid moods above.

In addition to the dictum de omni, proofs for valid de dicto syllogisms require our Axioms 1 and 2 (Section 3 above), and the following rule:

Rule d If $\square x$ and $\square y$ then $\square(x$ and $y)$.

That this indeed should be a rule can be seen by looking at the truth claims of de dicto apodeictic statements. The claim of ' $\square x$ ' is ' $\cap d$ is $[x]$ '; that of ' $\square y$ ' is ' $\cap d$ is $[y]$ '. Now in Sommers's system the following is a rule (in [27] it is Law 13(ii) on p. 185 and Theorem 12 on p. 401):

$$
\text { If } X+A \text { and } X+B \text { then } X+\langle+A+B\rangle \text {. }
$$

So, by this rule, if $\cap d$ is $[x]$ and $\cap d$ is $[y]$, then $\cap d$ is $\langle[x]+[y]\rangle$. And ' $\cap d$ is $\langle+[x]+[y]\rangle$ ' (i.e., 'the intersection of domains is both $[x]$ and $[y]$ ') is the truth claim of ' $\square(x$ and $y)$ '. 
The following are proofs for the first three moods above.

Proof of 1.d: 1. $\square(-M+P)$

premise

2. $\square(-S+M)$

premise

3. $\square(+[-M+P]+[-S+M])$

4. $\square(-S+P)$

1, 2, Rule d

Proof of 2.d: 1. $\square(-M+P)$

2. $\square(-S+M)$

3. $\square(+[-M+P]+[-S+M])$

4. $\square(-S+P)$

5. $-S+P$

3, dictum de omni

Proof of 3.d: $\begin{array}{r}\text { 1. } \square(-M+P) \\ \text { 2. } \square(-S+M)\end{array}$

3. $\square(+[-M+P]+[-S+M])$

4. $\square(-S+P)$

premise premise

5. $-S+P$

6. $\diamond(-S+P)$

1, 2, Rule d

3, dictum de omni

4, Axiom 1

premise

premise

1,2 , Rule d

3, dictum de omni

4, Axiom 1

5, Axiom 2

Proofs similar to 1.r-3.r and 1.d-3.d are available for modal versions of all valid moods. All that is needed to be added to the proof theory of the assertoric syllogistic are a small number of very simple principles (our four axioms and Rule d). A system of greatly extended inference power is purchased for this small price. In fact, even the kinds of mixed valid syllogisms mentioned by Ockham are provable in our system. For example, we can easily prove:

$$
\begin{gathered}
\square(-M+P) \\
-S+M \\
\hline-S+\diamond P .
\end{gathered}
$$

Proof: $1 . \square(-M+P)$

premise

2. $-S+M$

premise

3. $-M+P$

1, Axiom 1

4. $-S+P$

5. $-S+\diamond P$

2, 3, dictum de omni

4, Axiom 4

It has probably been noticed that apodeictic conclusions follow only from pairs of apodeictic premises. Indeed, we can think of our general forms of statements as arranged in order of "strength" (where one sentence is stronger than a second if and only if the first entails the second but the second does not entail the first). Our four axioms guarantee the following list of forms in descending order of strength.

$$
\begin{gathered}
\square x \\
x \square \\
x \\
x \diamond \\
\diamond x .
\end{gathered}
$$

Within each of these levels we have further levels determined by standard subalternation. Thus: 


$\square \mathrm{a} / \mathrm{e}$
$\square \mathrm{i} / \mathrm{o}$
$\mathrm{a} / \mathrm{e} \square$
$\mathrm{i} / \mathrm{o} \square$
$\mathrm{a} / \mathrm{e}$
$\mathrm{i} / \mathrm{o}$
$\mathrm{a} / \mathrm{e} \diamond$
$\mathrm{i} / \mathrm{o} \diamond$
$\diamond \mathrm{a} / \mathrm{e}$
$\diamond \mathrm{i} / \mathrm{o}$.

The relative orders of strength for statements is inversely proportional to the relative sizes of the domains which they denote-the stronger the statement the smaller the domain. For, given a domain $d$,

$$
\cap d \subseteq \cap d^{*} \subseteq d \subseteq \cup d^{*} \subseteq \cup d .
$$

All this suggests that a necessary condition for the validity of any syllogism is that the conclusion cannot exceed any premise in strength. The Scholastics called this the peiorem rule (peiorem semper sequiter conclusio partem).

Another necessary condition is suggested by the fact that no syllogism with both premises de dicto problematic or one premise and the conclusion de dicto problematic is valid. Let ' $\nabla p$ ' and ' $\nabla q$ ' be two of the sentences of a syllogism. Their truth claims are ' $\cup d$ is $[p]$ ' and ' $U d$ is $[q]$ ' respectively. Now ' $\cup d$ is $[p]$ ' is satisfied whenever at least one domain is $[p]$; and ' $U d$ is $[q]$ ' is satisfied whenever at least one domain is $[q]$. But there is nothing which guarantees that there is any common domain which is both $[p]$ and $[q]$. In other words, though ' $\nabla p$ ' and ' $\nabla q$ ' are true just in case ' $p$ ' is true in some domain and ' $q$ ' is true in some domain, there is no guarantee that there is a domain in which they are both true. For example, both 'Possibly some horse is winged' and 'Possibly every winged object is a bird' are true. Yet it need not follow that possibly some horse is a bird. This suggests that we demand of any valid syllogism that the respective domains of each of the sentences stand in the inclusion relation to one another. ${ }^{8}$ Since this need not hold for the domains which ground the truth of any pair of de dicto problematic statements, we cannot permit more than one such statement within any syllogism. This, along with the peiorem rule, guarantees that if there is such a statement in a syllogism it must be the conclusion. If we add this new condition and the peiorem rule to the standard conditions for syllogistic validity we get the following five conditions, which are individually necessary and jointly sufficient for the validity of a syllogism.

1. The middle term must be distributed at least once.

2. Any term distributed in the conclusion must be distributed in the premises.

3. The number of particular conclusions must not be exceeded by the number of particular premises.

4. The conclusion must not exceed any premise in strength. (peiorem)

5. The number of de dicto problematic sentences in a syllogism must not exceed one. ${ }^{9}$ 
We conclude with a final cautionary note. Sommers has succeeded in building a version of syllogistic which matches (and may exceed) the standard predicate calculus in naturalness, expressive power, and simplicity. We have attempted here to indicate one important direction in which his logic can be extended. That such an augmented syllogistic will have greatly expanded inference power is undeniable. Whether our own version of this extension is the only or best one is far from certain. We have offered only a glimpse of a new modal syllogisticmuch more can and must be done.

\section{NOTES}

1. Aristotle's remarks allow for corresponding Darii versions as well.

2. Bochénski ([1], p. 58) took Barbara I(d) to be valid. But he assimilated de dicto to de re modality.

3. There are restrictions on doing so however. See [27], Chapter 14; and [3].

4. For more on the status of singular in the new syllogistic see: [25], Chapter 6 of [27], [2], [5], [24], [29].

5. See De Interpretatione, $22 \mathrm{~b} 11$ and 23a21ff, for Aristotle's statements of these.

6. Figure 5 should be seen as an improvement on McCall's Table 6 in [9], p. 35.

7. McCall ([9], p. 46) claimed that 333 are valid when modality is restricted to de dicto or to de re.

8. This is actually part of a general condition on all discourse, requiring that there be some specifiable domain common to or included in all statements constituting that discourse. We eannot "mix domains". From facts about Pegasus and facts about Kentucky Derby: winners we cannot draw conclusions about flying horses at the Derby.

9. Łukasiewicz took syllogisms with at least a de dicto problematic conclusion and major to be valid. See [8], p. 193.

\section{REFERENCES}

[1] Bochénski, I.M., Ancient Formal Logic, North-Holland Publishing Company, Amsterdam, 1968.

[2] Englebretsen, G., "Singular terms and the syllogistic," The New Scholasticism, vol. 54 (1980), pp. 68-74.

[3] Englebretsen, G., "Quadratum Auctum," Logique et Analyse, vol. 107 (1984), pp. 309-325.

[4] Englebretsen, G., "Semantic considerations for Sommers' logic," Philosophy Research Archives, vol. 11 (1985), pp. 281-318.

[5] Englebretsen, G., "Singular/general," Notre Dame Journal of Formal Logic, vol. 27 (1986), pp. 104-107.

[6] Englebretsen, G., The New Syllogistic, Peter Lang Publishing Company, New York, 1987.

[7] Kneale, M. and W. Kneale, The Development of Logic, Clarendon Press, Oxford, 1962. 
[8] Lukasiewicz, J., Aristotle's Syllogistic, Clarendon Press, Oxford, 1957.

[9] McCall, S., Aristotle's Modal Syllogisms, North-Holland Publishing Company, Amsterdam, 1963.

[10] Noah, A., "Predicate-functors and the limits of decidability in logic," Notre Dame Journal of Formal Logic, vol. 21 (1980), pp. 701-707.

[11] Noah, A., "Quine's version of term logic and its relation to TFL," Appendix F of [27].

[12] Noah, A., "Predicate functors and English pronouns," forthcoming.

[13] Quine, W.V., "Concepts of negative degree," Proceedings of the National Association of Science, vol. 22 (1936), pp. 40-45.

[14] Quine, W.V., "Toward a calculus of concepts," The Journal of Symbolic Logic, vol. 1 (1936), pp. 2-25.

[15] Quine, W.V., "Logic based on inclusion and abstraction," The Journal of Symbolic Logic, vol. 2 (1937), pp. 145-152.

[16] Quine, W.V., "Eliminating variables without applying functions to functions," The Journal of Symbolic Logic, vol. 24 (1959), pp. 324-325.

[17] Quine, W.V., "Variables explained away," Proceedings of the American Philosophical Society, vol. 104 (1960), pp. 343-347, reprinted as pp. 227-235 in [19].

[18] Quine, W.V., "Logic as a source of syntactical insights," Proceedings of Symposia in Applied Mathematics, vol. 12 (1961), pp. 1-5; reprinted as pp. 44-49 in [22].

[19] Quine, W.V., Selected Logic Papers, Random House, New York, 1966.

[20] Quine, W.V., "Algebraic logic and predicate functors," Bobbs-Merrill, Indianapolis, 1971; revised and reprinted as pp. 283-307 in [22].

[21] Quine, W.V., "The variable” (1972), pp. 272-282 in [22].

[22] Quine, W.V., The Ways of Paradox and Other Essays, Revised and Enlarged Edition, Harvard University Press, Cambridge, Massachusetts, 1976.

[23] Quine, W.V., "Predicate functors revisited," The Journal of Symbolic Logic, vol. 46 (1981), pp. 649-652.

[24] Slater, B. H., "Singular subjects," Dialogue, vol. 18 (1979), pp. 362-372.

[25] Sommers, F., "Do we need identity?," Journal of Philosophy, vol. 66 (1969), pp. 499-504.

[26] Sommers, F., “The calculus of terms," Mind, vol. 79 (1970), pp. 1-39.

[27] Sommers, F., The Logic of Natural Language, Clarendon Press, Oxford, 1982.

[28] Sommers, F., "Truth and existence," pp. 299-304 in [6].

[29] Wald J. D., "Geach on atomicity and singular propositions," Notre Dame Journal of Formal Logic, vol. 20 (1979), pp. 285-294. 